\title{
Mortalidad por causas externas en tres ciudades latinoamericanas: Córdoba (Argentina), Campinas (Brasil) y Medellín (Colombia), 1980-2005*
}

\author{
Doris Cardona** \\ Enrique Peláez $z^{\star \star \star}$ \\ Tirza Aidar**** \\ Bruno Ribotta**** \\ María Franci Alvarez $z^{* * * * *}$
}

\begin{abstract}
Objetivo. Contribuir a los estudios sobre mortalidad por causas violentas en la América Latina a través de la análisis comparativa de los patrones y tendencias reciente de las muertes por causas externas en tres contextos urbanos regionales. Métodos. Estudio descriptivo de fuente secundaria, utilizando estadísticas vitales del período 1980 a 2005, de Córdoba (Argentina), Campinas (Brasil), y Medellín (Colombia) en tres períodos entre 1980 y 2005. Destacan los siguientes grupos de causas: homicidios por armas de fuego y otros, accidentes de transportes, suicidios y de intención no determinada; desagregado por edad y sexo, con tasas calculadas con media de trienios próximos a los años censales. Resultados. Los niveles de Medellín aventajan notablemente los de Campinas y Córdoba, en todas las causas estudiadas y en todas ellas son los hombres jóvenes los que ponen la mayor cuota de muertes. Los niveles de Campinas duplican a los de Córdoba, sobre todo en los homicidios y accidentes de tránsito, pero en los suicidios las tasas cordobesas duplican a las de Campinas. Para Medellín las tasas son máximas en torno de 1990, contrario de las otras ciudades donde la tendencia es creciente entre 1980 y 2000, y decreciente entre 2001 y 2005. Conclusiones. La disponibilidad de datos sobre mortalidad con calidad permite comparaciones sobre la salud de las poblaciones estudiadas. Al comparar la mortalidad por causas externas, se evidencian diferencias en el nivel pero no tanto en el comportamiento por edad y sexo; a pesar que se trata de ciudades con algunas características similares por lo porte, además son centros universitarios y de desarrollo industrial de importancia en cada país. Los resultados sugieren que factores socioeconómicos y demográficos no son suficientes para explicar la gran diferencia en las cantidades observadas.
\end{abstract}

Palabras claves: Mortalidad. Causas externas. Violencia. Calidad de la información.

\footnotetext{
* Trabajo presentado en lo XVI Encontro Nacional de Estudos Populacionais, Caxambu, 29 septiembre a 3 octubre del 2008. Apoyado por CAPES - Programa de Centros Asociados de Pos-Grado, Brasil-Argentina, programas de pos-grado en Demografía del IFCH y NEPO/UNICAMP, y CEA/UNC.

** Magíster en Epidemiología, Magíster en Salud Pública y Candidata a Doctor en Demografía, Docente Investigadora en Universidad de Antioquia y Universidad CES.

*** Doctor en Demografía, Demógrafo de la CONICET - Universidad Nacional de Córdoba.

**** Doctor en Demografía, Docente Investigadora del IFCH e NEPO - Universidade Estadual de Campinas.

***** Magister en Demografía, docente del Centro de Estudios Avanzados - CEA, de la Universidad Nacional de Córdoba - UNC.

${ }_{\star \star \star \star \star \star \star}$ Docente Investigadora del Centro de Estudios Avanzados - CEA, de la Universidad Nacional de Córdoba UNC y Universidad Nacional de Villa María.
} 


\section{Introducción}

En 1997, la OMS declara que, aunque la violencia sea tan antigua como el mundo, los nuevos hechos observados, juntamente con el crecimiento de su incidencia en los indicadores sanitarios de las últimas décadas, la transforman en un problema de salud pública regional (OPS, 1998). Asimismo, se ha destacado que la violencia

\begin{abstract}
[...] afecta a toda la población en la región de las Américas; sin embargo, en condiciones de pobreza y desventaja social, algunos sectores sociales resultan especialmente amenazados. Los hombres jóvenes se ven afectados primordialmente por enfrentamientos armados, desajustes sociales y laborales y situaciones de pobreza e inequidad. Es importante destacar que las situaciones de pobreza e inequidad afectan a hombres, mujeres y niños por igual, pero los homicidios se caracterizan por afectar principalmente a los hombres jóvenes. Ellos son las principales víctimas y agentes de violencia homicida. En la mitad de los países de la región con más de un millón de habitantes el homicidio constituye la segunda causa de muerte de varones entre 15 y 24 años de edad. (OPS, 1998)
\end{abstract}

En el año 2002 se registraron en el mundo 45 millones de muertes de personas de 15 años o más y de ellas 32 millones se debieron a enfermedades no transmisibles y 4,5 millones a traumatismos ( $70 \%$ muertes masculinas y afectan principalmente a la población de 15 a 44 años). Los traumatismos pueden ser accidentales o intencionales, los últimos incluyen las autolesiones, el suicidio y los actos de violencia y de guerra, representan una parte cada vez mayor de la carga, sobre todo entre los adultos jóvenes económicamente productivos. En los países desarrollados, los suicidios acaparan la proporción más importante de la carga atribuible a los traumatismos intencionales, mientras que en las regiones en desarrollo son la violencia y la guerra las que ocupan el primer lugar (OMS, 2003).

Latinoamérica se caracteriza por ser la región con mayor inequidad en la distribución del ingreso. Asimismo, los procesos de urbanización de las últimas décadas generaron grandes concentraciones de población con tendencia a la segregación espacial, convirtiendo a las ciudades en un continuo de espacios cerrados y villas de emergencia. Esta situación se transforma en un escenario propicio para la violencia en sus múltiples manifestaciones, que frecuentemente conducen a la muerte de los diferentes actores.

Al mismo tiempo que las transformaciones políticas, socioeconómicas y del mercado de trabajo afectan a los principales y mayores centros urbanos del mundo, y que el crecimiento de la criminalidad constituye en general una preocupación de diversos sectores de la sociedad contemporánea, es preciso considerar la complejidad del fenómeno y sus especificidades en los diferentes contextos regionales. Por ejemplo, cuando se analizan las tasas de mortalidad por homicidios en el año 2000, las diferencias son extremadamente evidentes entre los países de América Latina, variando de 65 muertes por cada 100 mil habitantes en Colombia, país con un largo periodo de guerra civil y acción paramilitar, a poco más de 5 homicidios por cada 100 mil habitantes en Argentina. En Brasil, este mismo indicador alcanzó a 27 muertes por 100 mil para el mismo periodo (AIDAR; SOARES, 2006).

Las diferencias entre las principales ciudades de América son también importantes ${ }^{1}$; hacia el año 2000 , las mayores tasas de mortalidad por homicidios se observan en Recife (Brasil), Medellín y Cali (Colombia), Guatemala (Guatemala) y San Salvador (El Salvador), con más de 90 muertes por cada 100 mil habitantes. En el mismo periodo, los menores índices de homicidios fueron observados en las ciudades de México (México), Quito (Ecuador), Panamá (Panamá), Miami (EEUU), Santiago de Chile (Chile), Buenos Aires y Córdoba (Argentina), con tasas menores a 20 muertes por cada 100 mil habitantes, destacándose las dos últimas ciudades por los índices mínimos, de 5 homicidios por cada 100 mil habitantes.

\footnotetext{
1 "Córdoba tiene una baja tasa anual de homicidios. La ciudad se encuentra muy lejos de los índices de las capitales latinoamericanas más inseguras". Disponible en: <http://www2.lavoz.com.ar/nota.asp?nota_id=93921>.
} 
No obstante la heterogeneidad, existe consenso entre los estudios demográficos en cuanto al crecimiento de las muertes violentas, y más aún, sobre el incremento de la sensación de inseguridad frente a los índices de homicidios. No se verifica el mismo acuerdo en la explicación de los principales determinantes del fenómeno. En las comparaciones entre regiones de un mismo país, o en investigaciones internacionales, los resultados no siempre son conclusivos respecto al papel de la pobreza, la desigualdad socio-económica o el deterioro de las condiciones de vida en los centros urbanos, sea desde la perspectiva de los individuos que responden de determinada forma ante las restricciones de orden material, o desde el punto de vista del contexto social o cultural que regula el proceso de decisión individual. Como destacan Freitez y Romero (2004), el debate es amplio y la forma o intensidad de la contribución de los factores individuales, familiares o socioculturales, dependerá del contexto y del momento histórico (BRICEÑO-LEÓN, 2004 apud FREITEZ; ROMERO, 2004).

Para el caso de Brasil, simultáneamente al deterioro de la calidad de vida en los grandes centros urbanos, algunos investigadores han destacado el importante papel de la expansión del crimen organizado, el tráfico de drogas y armas de fuego, en adición a la fragilidad para controlar, o asimismo la connivencia, de algunos sectores institucionales (ADORNO; CARDIA, 2002; ZALUAR, 1999).
En términos generales, la mortalidad en Colombia se ha mantenido constante durante los últimos 13 años, pero los eventos conocidos como de causalidad externa relacionados con la violencia y los accidentes de tránsito han registrado un incremento notable, constituyéndose en uno de los principales problemas de salud pública de la actualidad. Entre todos los países de América, Colombia tiene uno de los más altos índices de violencia, estimándose que el $15 \%$ se debe a causas políticas y el $85 \%$ a conflictos cotidianos (COLOMBIA. MINISTERIO DE SALUD, 2002).

El informe de la Dirección Nacional de Política Criminal de Argentina elaborado basándose en datos de la Organización Panamericana de la Salud (OPS) indica que los "[...] diversos criterios con los que se relevan los datos de la delincuencia dificultan, si no impiden, realizar comparaciones legítimas entre países en la mayoría de los delitos. De allí que sólo se hace referencia a los pocos tipos de criminalidad cuya contabilidad internacional no ofrece problemas"2.

Dicha información brinda la oportunidad de comparar la situación en Argentina, Brasil y Colombia en la década pasada. En todo el periodo, las tasas de homicidios dolosos de Brasil duplicaron los de Argentina, a su vez las cifras brasileras fueron triplicadas y hasta en algunas oportunidades cuadruplicadas por las cifras de Colombia, lo cual plantea el desafío de analizar la información del contexto como forma de comprender las diferencia (Cuadro 1).

CUADRO 1

Tasa de homicidios

Brasil, Argentina y Colombia - 1990-2000

Por 1000.000 habitantes

\begin{tabular}{|c|c|c|c|c|c|c|c|c|c|c|c|}
\hline \multirow{2}{*}{ País } & \multicolumn{11}{|c|}{ Año } \\
\hline & 1990 & 1991 & 1992 & 1993 & 1994 & 1995 & 1996 & 1997 & 1998 & 1999 & 2000 \\
\hline Brasil & 18,6 & 17,5 & 15,6 & 16,7 & 17,5 & 19,3 & 24,0 & 25,0 & 26,0 & 25,0 & 19,7 \\
\hline Argentina & 7,5 & 7,5 & 9,0 & 7,5 & 7,6 & 7,8 & 8,5 & 9,0 & 7,3 & 7,3 & 7,2 \\
\hline Colombia & - & - & 71,4 & 68,7 & 63,3 & 60,8 & 69,4 & 65,0 & 54,0 & 59,0 & 65,0 \\
\hline
\end{tabular}

Fuente: Organización Panamericana de la Salud citada por la Dirección de Política Criminal de Argentina.

\footnotetext{
${ }^{2}$ Comparación de homicidios dolosos en países americanos por regiones. Disponible en: <http://wwwpolcrim.jus.gov.ar> Actualización 13 de febrero de 2006.
} 
Paes y Silva (2000) alertan sobre el agravamiento de la mortalidad por causas externas en Brasil, el cual es muy notorio entre los años 1980 y 1990, y particularmente entre la población joven. Del mismo modo, en un estudio auspiciado por la UNESCO, Waiselfisz y Athias (2005) da cuenta del incremento, entre 1980 y 1996, de las tasas de mortalidad de los jóvenes brasileños y de la participación de las muertes por causas externas dentro del total de las defunciones de 15 a 24 años, de 53\% a 67\%. En ese trabajo se ha destacado también que la mortalidad por homicidios y otras formas de violencia casi se duplica entre los jóvenes que residen en las capitales estatales y en las regiones metropolitanas respecto al promedio nacional.

Un estudio realizado en la Argentina, que considera los años 1980, 1991 y 1995, encontró que los varones de todas las edades poseen un riesgo mayor de defunciones por violencia que las mujeres, y esta diferencia se hace más evidente en las edades de 15 a 24 años. El mismo trabajo identificó un aumento considerable en las causas agrupadas como Lesiones en que se ignora si fueron accidental o intencionalmente infligidas; en el periodo estudiado, se triplicó la tasa de mortalidad en los varones y se duplicó en las mujeres, y dentro de este grupo la causa simple que más aportó al crecimiento, además de la ocasionada por armas de fuego, es la que corresponde a otros medios y los no especificados.

En una investigación realizada por Serfaty et al. (2003) señala que en Argentina las tasas de mortalidad de jóvenes y adolescentes aumentaron un 6\% entre 1990 y 2001, debido al aumento de las muertes masculinas. Las muertes violentas pasaron del $38 \%$ de las defunciones de jóvenes y adolescentes en 1990 al $50 \%$ en el 2001. Otro antecedente que aborda la problemática de la violencia en Argentina, se observó que la tendencia de los accidentes durante el periodo 1991-2000 es decreciente, los accidentes de vehículo de motor continúan como la principal causa de muerte; por otro lado, las agresiones tienden a ser un problema creciente, así como los suicidios que han tenido un aumento importante en población masculina (ALVAREZ, 2002).

En una comparación de la mortalidad entre Brasil y Venezuela (FREITEZ e ROMERO, 2004), se constata no existiría una relación directa entre pobreza y violencia, y que nos dos países las tasas más altas de homicidios se registran no en aquellas entidades federales más pobres, sino en las que hay más contrastes sociales entre ricos y pobres. Venezuela, Colombia y Brasil son los países que registran las más altas y crecientes tasas de mortalidad juvenil por causas violentas entre 1980 y 1997. Argentina está ubicada dentro de los países con tasas moderadas.

En razón de los puntos mencionados, este trabajo busca contribuir a los estudios sobre mortalidad por causas violentas en la América Latina a través de la análisis comparativa de los patrones y tendencias reciente de las muertes por causas externas en los tres contextos urbanos regionales.

\section{Las ciudades de Córdoba (Argentina), Campinas (Brasil) y Medellín (Colombia)}

Córdoba es la capital de la provincia homónima, ubicada en el centro de la Argentina. La ciudad se localiza en el departamento Capital, y constituye el componente principal del aglomerado Gran Córdoba, segundo en extensión del país. Se caracteriza por constituir un centro educativo, industrial (fundamentalmente automotriz) y de servicios. Córdoba registró una población total de 1.284.582 habitantes en el censo 2001, sendo casi en su totalidad población urbana. La ciudad comparte a grandes rasgos las tendencias de las áreas más urbanizadas de la Argentina, entre las que se destacan una reducción de su ritmo de crecimiento medio anual y un creciente proceso de envejecimiento. Según la estructura etárea, el porcentaje de población menor de 14 años representó el $28,7 \%$ de la población total en el año 1991, mientras que en el año 2001, el porcentaje descendió al 26,1\%. Concomitantemente, la población de 60 años y más, se incrementó en un $11,1 \%$ entre los años 
mencionados (pasando de $11,7 \%$ al $13 \%$, respectivamente).

La localidad de Córdoba Capital constituye un centro educativo de importancia nacional, fundamentalmente a escala universitaria. Este hecho representa tanto un paliativo al proceso de envejecimiento mencionado (por inmigración de estudiantes), como uno de los tantos factores que explican el incremento del nivel educativo de su población, donde se destaca la reducción de la población no escolarizada de 11 a 17 años, y el incremento de la escolaridad media de los mayores de 25 .

No obstante lo anterior, desde mediados de los '90 la crisis del empleo en la Argentina, y en particular, la retracción de la industria automotriz de la ciudad, han representado que la inserción laboral afecte también a otros sectores de la población, y en ésta, a la más desfavorecida socio-económicamente. Las tasas de desempleo de la población de 20 a 24 años se incrementan desde un $12,3 \%$ en el año 1991 a $23 \%$ en el año 2001, y la correspondiente a 25-49 años, de 2,9\% en la primera fecha a $14 \%$ en la segunda. Finalmente, el desempleo de la población de 50 y más años, pasa de $0,6 \%$ en el año 1991, al 10,7\% en el año 2001.

La ciudad de Campinas registró en el censo de población del año 2000 , población de 969.396 habitantes. Cunha e Oliveira (2001, p.2-3), afirman que este municipio mostró una estrecha semejanza con lo observado en otras metrópolis brasileñas, en relación con las importantes tasas de crecimiento demográfico, junto con la periferización del crecimiento físico-territorial. Desde la década del 1970 se observa un flujo migratorio desde la sede metropolitana, que provoca una expansión más allá de sus límites.

Este proceso tiene su correlato en varias de las ciudades menores de la región metropolitana de Campinas, donde el sistema de carreteras y el dinamismo económicoregional representan ventajas comparativas que favorecen su crecimiento. La ciudad de Campinas presenta una desaceleración de su crecimiento poblacional, y un aumento de la participación de la población mayor de 60 años. También es un importante centro educacional de la enseñanza superior, con una de las más importantes universidades públicas brasileras (Universidad Estadual de Campinas) y una variada oferta de instituciones privadas. En general, los indicadores educacionales de sus habitantes son más elevados que la media nacional y presentan en el periodo analizado incremento de la cantidad de niños y jóvenes que asisten a la escuela, así como de los niveles de escolaridad promedio de la población adulta. Entretanto, es importante destacar que, de manera semejante a Córdoba, existen diferenciales relevantes que dependen del grupo socio-espacial considerado (AIDAR, 2003). Se verifica para el año 2000, en varias áreas de la ciudad, que la proporción de menores de 11 a 14 años que no asisten a la escuela supera al 14\% (AIDAR; SOARES, 2006).

Medellín es la capital del departamento de Antioquia, ubicada en Colombia. Según el censo 2005, la tasa de crecimiento anual es de 14,4 por mil, la población total fue de 2.219.861 habitantes, distribuidos entre el $46,7 \%$ masculina y el $53,7 \%$ femenina; la estructura por edad muestra a una población con el $24,9 \%$ de menores de 15 años, el 9,1 es población adolescente de 15 a 19 años, el $29,5 \%$ son mujeres en edad fértil y, el 10,4\% de 60 y más años (COLOMBIA. DEPARTAMENTO ADMINISTRATIVO NACIONAL DE ESTADÍSTICAS, 2007).

Como las otras, Medellín cuenta con un amplio sistema educativo formal; primario, secundario y universitario. El $78 \%$ de los alumnos estudian en escuelas y colegios del sector público; es la ciudad universitaria del país por excelencia con más de 130 mil estudiantes en educación superior, distribuidas en 35 instituciones públicas y privadas.

En cuando a los indicadores demográficos las tres ciudades comparadas presentan estructuras poblacionales similares. Indicadores sobre empleo reflejan las modificaciones en el mercado de trabajo regional, con significativo aumento de los índices de desempleo en Córdoba y Campinas, principalmente entre los más jóvenes.

\section{Materiales y métodos}

Estudio descriptivo de fuente secundaria. Las fuentes de información utilizadas 
son los Censos Nacionales de Población de Argentina, Brasil y Colombia del período 1980 a 2005 y las estadísticas vitales de las ciudades de Córdoba, Campinas y Medellín para el examen de las causas de muerte agrupadas en: agresiones, suicidios, accidentes de transporte, accidentes y otros eventos no determinados y otras causas externas, separados si fueron infringidos con armas de fuego o no (Anexo 1).

El análisis es presentado por edad y sexo para tres momentos: los trienios de 1990 a 1992, de 1999 a 2001 (o de 2000 a 2002) y de 2003 a 2005. Cuando resulta posible, se incluyen datos correspondientes a la década de 1980-1989. Las tasas de mortalidad fueron calculadas con media de trienios próximos a los años censales.

Finalmente se valora la comparabilidad de datos y fuentes de información entre Argentina, Colombia y Brasil, de las estadísticas vitales para estudios de mortalidad por causas externas.

\section{Resultados}

En lo Cuadro 2 observa-se que en Córdoba los accidentes con arma de fuego (a) y las lesiones en las que se ignora si fueron accidentales $o$ intencionalmente infligidas con armas de fuego (b), representaban cerca de $18,1 \%, 15,0 \%$ y $12,8 \%$ de las defunciones por causas externas, en los trienios 1990/1992, 2000/2002 y de 2003/2005, respectivamente. Por otro lado, el porcentaje de muertes violentas clasificadas como otras lesiones en las que se ignora si fueron accidentales o intencionalmente inflingidas (g), incrementan su representación porcentual desde el $27,6 \%$ entre $1990-1992$, al $40,3 \%$ en 2003-2005. Curiosamente, el porcentaje de accidentes no determinados (h), desciende desde un $10,4 \%$ en la primera fecha, a menos en un $1 \%$ en la segunda.

En Campinas, los accidentes y los eventos con armas de fuego con intención no determinada, (grupos a y b), representaron solamente $1,8 \%, 4,9 \%$ y $2,1 \%$ de muertes por causas externas en los trienios 1990/1992, 1999/2001 y de 2003/2005, respectivamente. Para los últimos años del período analizado, como los datos de Córdoba, en la ciudad brasilera los demás eventos con intención no determinada (grupo g) constituyen las causas externas sin definición de mayor representatividad y con aumento de $8,0 \%$ a $15,8 \%$ entre los trienios $1990 / 1992$ y 2003/2005, respectivamente (Cuadro 2).

En Medellín, los homicidios con arma de fuego (c) bajaron del $77,1 \%$ del total de defunciones violentas en el trienio 1990/1992 a $48,8 \%$ en el $2003 / 2005$, así mismo los accidentes de transportes que van en aumento del $6,6 \%$ a $12,4 \%$ y luego $20,3 \%$ en los tres períodos estudiados, y el tercer grupo de causas fueron los otros homicidios (grupo d) que estaba en 9,7\% entre 1990/1992 bajó a $7,6 \%$ en $1999 / 2001$ al $12 \%$ en el último período. Llama la atención el bajo porcentaje de eventos con intención no determinada $y$ de accidentes no determinados ( $b, g, h$ ) (Cuadro 2).

Como puede apreciarse, a lo largo del periodo considerado, el comportamiento de las causas externas en la ciudad de Medellín se diferencia notablemente de las ciudades de Campinas y Córdoba, presentando porcentajes de defunciones por eventos con intención no determinada inferiores al 1\%, y accidentes no determinados que apenas superan dicha cifra. Ante estos resultados y el crecimiento de la importancia de las armas de fuego en las muertes violentas, en el análisis siguiente se considera también la tendencia de las causas externas no definidas, en especial aquellas efectuadas con armas de fuego, según causas descritas en el Cuadro 2, que serán agrupados a los homicidios formando el total de las agresiones.

Entonces, cuando se incorporan a los homicidios $(c+d)$, las muertes clasificadas como accidentes con arma de fuego (a) y las lesiones en las que se ignora si fueron accidentales o intencionalmente infligidas, hechas con arma de fuego (b), se verifica que estos representan cerca de $22,5 \%, 23,7 \%$ y $19,7 \%$ de las defunciones por causas externas en Córdoba, para los años centrados en 1991, 2000 y 2004, respectivamente. En Campinas, esos mismos grupos de causas representaron $28,1 \%, 57,2 \%$ y $46,8 \%$ en los mismos años respectivamente, mientras que en Medellín la concentración de las muertes 
CUADRO 2

Distribución de las defunciones por causas externas seleccionadas

Córdoba (Argentina), Campinas (Brasil) y Medellín (Colombia) - 1990-2005

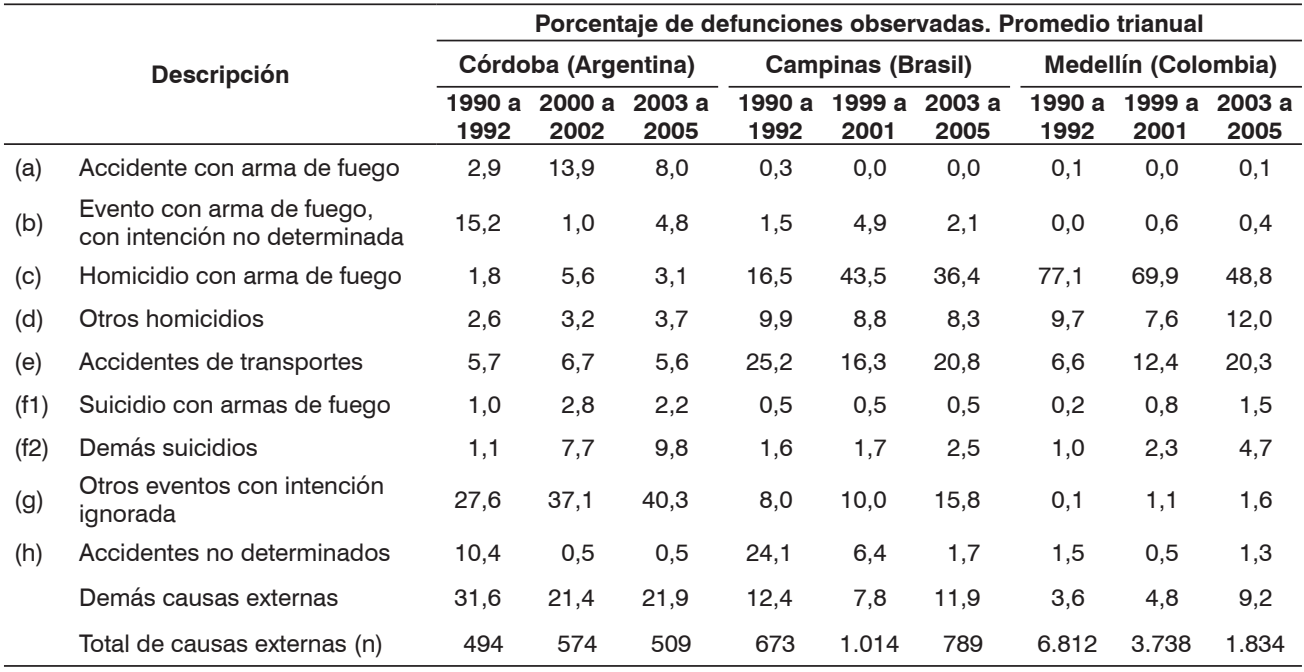

Fuente: IBGE, Censos Nacionales de Brasil; Sistema de Informaciones de Mortalidad, Ministerio de la Salud de Brasil. INDEC Censos Nacionales de Población de Argentina. Dirección Nacional de Estadísticas e Información en Salud, Ministerio de Salud de Argentina. DANE, Censos Nacionales de Población y Registros de Defunciones de Colombia. Elaboración propia.

externas en agresiones fue mucho más marcada ya que estas significaron el $86,9 \%$, el $78,2 \%$ y el $61,3 \%$ respectivamente.

El elevado porcentaje de defunciones por eventos de intención no determinada y otras causas externas distorsiona el análisis que puede hacerse para cada uno de los grupos debiendo ser prudentes en las conclusiones obtenidas. En Medellín la alta concentración en agresiones hace que las muertes externas mal definidas pierdan importancia en su impacto sobre el total y mismo para la comparación con Córdoba e Campinas.

\section{Niveles y tendencias de la mortalidad}

En todo el periodo analizado, el nivel de mortalidad por causas externas de Medellín supera considerablemente al observado en Córdoba y Campinas (Cuadro 3 y Figura 1). Entre los años 1990-1992, la tasa de Medellín multiplica por diez a la tasa de Córdoba y por cinco a la de Campinas. Hacia el periodo siguiente (años 2000-2002), las diferencias entre las ciudades se mantienen, pero menos intensamente: la tasa de mortalidad por causas externas de Medellín prácticamente quintuplica a la de Córdoba y duplica a la de Campinas. Esta modificación se debe a una reducción considerable en el nivel de MedeIlín (-53,7\%), mientras que la mortalidad por causas externas de Córdoba y Campinas experimenta aumentos de $3,7 \%$ y $25,1 \%$ respectivamente. Hacia los años 2003-2005, se aprecia una reducción notable en el nivel de mortalidad por causas externas de Medellín (del $-55,8 \%$ en tan solo 4 años), que acerca su tasa a la de Campinas $(85,6$ por cien mil con respecto a 76,2 por cien mil), y reduce su brecha con Córdoba (prácticamente el doble de la tasa de esta última ciudad). Estas dos últimas ciudades también logran reducir su nivel de mortalidad por causas externas (-26,1 \% en Campinas y $-17,1 \%$ en Córdoba).

Mientras que Medellín mantiene la tendencia a la baja apreciada en los últimos periodos, Campinas y Córdoba revierten la tendencia al alza observada en los años 1990, obteniendo niveles más bajos que los iniciales.

Los patrones indicados anteriormente parecen en gran parte determinados por el 
CUADRO 3

Tasa de mortalidad y tipificada por edad, presentadas por trienios y causas externas seleccionadas Córdoba (Argentina), Campinas (Brasil) y Medellín (Colombia) - 1990-2005

Por 1000.000 habitantes

\begin{tabular}{|c|c|c|c|c|c|c|c|c|c|}
\hline \multirow[b]{2}{*}{ Grupos de causas } & \multicolumn{3}{|c|}{ Córdoba } & \multicolumn{3}{|c|}{ Campinas } & \multicolumn{3}{|c|}{ Medellín } \\
\hline & $\begin{array}{c}1990 \mathrm{a} \\
1992 \\
\end{array}$ & $\begin{array}{c}2000 a \\
2002\end{array}$ & $\begin{array}{l}2003 a \\
2005\end{array}$ & $\begin{array}{c}1990 \mathrm{a} \\
1992 \\
\end{array}$ & $\begin{array}{c}1999 \text { a } \\
2001\end{array}$ & $\begin{array}{l}2003 a \\
2005\end{array}$ & $\begin{array}{c}1990 \text { a } \\
1992\end{array}$ & $\begin{array}{c}1999 a \\
2001\end{array}$ & $\begin{array}{c}2003 a \\
2005\end{array}$ \\
\hline Agresiones (1) & 9,6 & 10,4 & 7,3 & 22,3 & 57,8 & 35,7 & 357,9 & 150,6 & 52,9 \\
\hline Suicidios & 0,9 & 4,6 & 4,3 & 1,8 & 2,2 & 2,2 & 5,3 & 6,0 & 5,3 \\
\hline Accidentes de transportes & 2,4 & 2,9 & 2,1 & 20,8 & 16,7 & 15,6 & 29,6 & 24,1 & 17,1 \\
\hline $\begin{array}{l}\text { Accidentes y otros eventos } \\
\text { no determinados ( } 2 \text { ) }\end{array}$ & 15,9 & 16,2 & 14,4 & 26,7 & 16,9 & 12,9 & 7,2 & 3,0 & 2,4 \\
\hline Otras causas externas & 12,5 & 8,7 & 7,4 & 9,9 & 8,2 & 9,0 & 16,6 & 9,3 & 7,7 \\
\hline Total causas externas & 41,2 & 42,8 & 35,5 & 81,5 & 102,0 & 75,3 & 416,6 & 193,0 & 85,4 \\
\hline Total de muertes & 674,5 & 746,0 & 735,9 & 608,1 & 607,4 & 582,0 & 916,8 & 650,7 & 533,6 \\
\hline$\%$ de causas externas & 6,1 & 5,9 & 5,0 & 13,1 & 17,2 & 13,3 & 46,9 & 29,6 & 15,9 \\
\hline
\end{tabular}

Fuente: IBGE, Censos Nacionales de Brasil; Sistema de Informaciones de Mortalidad, Ministerio de la Salud de Brasil. INDEC, Censos Nacionales de Población de Argentina. Dirección Nacional de Estadísticas e Información en Salud, Ministerio de Salud de Argentina. DANE, Censos Nacionales de Población y Registros de Defunciones de Colombia. Elaboración propia.

(1) Todos los homicidios, los accidentes con arma de fuego y otros eventos con arma de fuego con intención no determinada.

(2) Otros eventos con intención no determinada y accidentes no determinados.

tipo de causa externa involucrado (Cuadro 3 y Figura 1). En efecto, al desagregar los totales según un tipo de violencia (en los subgrupos de agresión, suicidio, accidentes de tránsito, otros accidentes y otras causas externas), se advierte:

La importancia de las agresiones en el nivel y evolución de la mortalidad por causas externas, cualquiera sea la ciudad involucrada. En Medellín, la mortalidad por agresiones representa $86,9 \%$ de la tasa de muerte por causas externas del periodo 1990-1992; 77,5\% del siguiente (1999-2001) y $60,9 \%$ del periodo final (2003-2005). En la ciudad de Campinas, estas proporciones son inferiores, pero explican notoriamente el incremento de la mortalidad por causas externas entre los años 1999 y 2001, y parte de su descenso hacia 2003-2005 (52,3\% y $44,7 \%$ respectivamente, de la tasa de muerte por causas externas). En Córdoba, las tasas de mortalidad por agresiones ocupan el tercer lugar (luego del grupo de accidentes y otros eventos no determinados, y de otras causas externas), pero presentan oscilaciones similares: incremento en el segundo periodo estudiado entre 2000 a $2002(22,7 \%)$ y reducción en el tercero de 2003 a 2005 (14,9\%).

La creciente importancia de la mortalidad por suicidio en las ciudades, cuyas tasas ubican nuevamente a Medellín en el primer lugar, pero a la ciudad de Córdoba en el segundo. En esta última ciudad, se observa que esta causa de muerte supera desde el año 2000 a la mortalidad por accidentes de transporte, hecho que no ocurre en las ciudades restantes.

La progresiva reducción de la mortalidad por accidentes de transporte (cuyos niveles iniciales más altos se encuentran en Medellín y Campinas, lugares en donde las tasas logran reducirse a 17,5 por cien mil y 15,8 por cien mil respectivamente). Una pauta similar también se observa a propósito de la mortalidad por otras causas externas y por accidentes y otros eventos no determinados, que alcanza las mayores reducciones en las mismas ciudades (primero en Campinas y luego en Medellín).

Por tal motivo puede señalarse que la elevada representatividad de la mortalidad por agresiones y la creciente importancia de la relacionada con el suicidio, contrasta con las reducciones sistemáticas en la mortalidad por accidentes y otras causas externas.

\section{Diferenciales por sexo y edad}

A través del cociente entre las tasas de mortalidad de varones y de mujeres se mide 
FIGURA 1

Evolución de las tasas de mortalidad por causas externas seleccionadas Córdoba (Argentina), Campinas (Brasil) y Medellín (Colombia) - 1980-2004 (1)
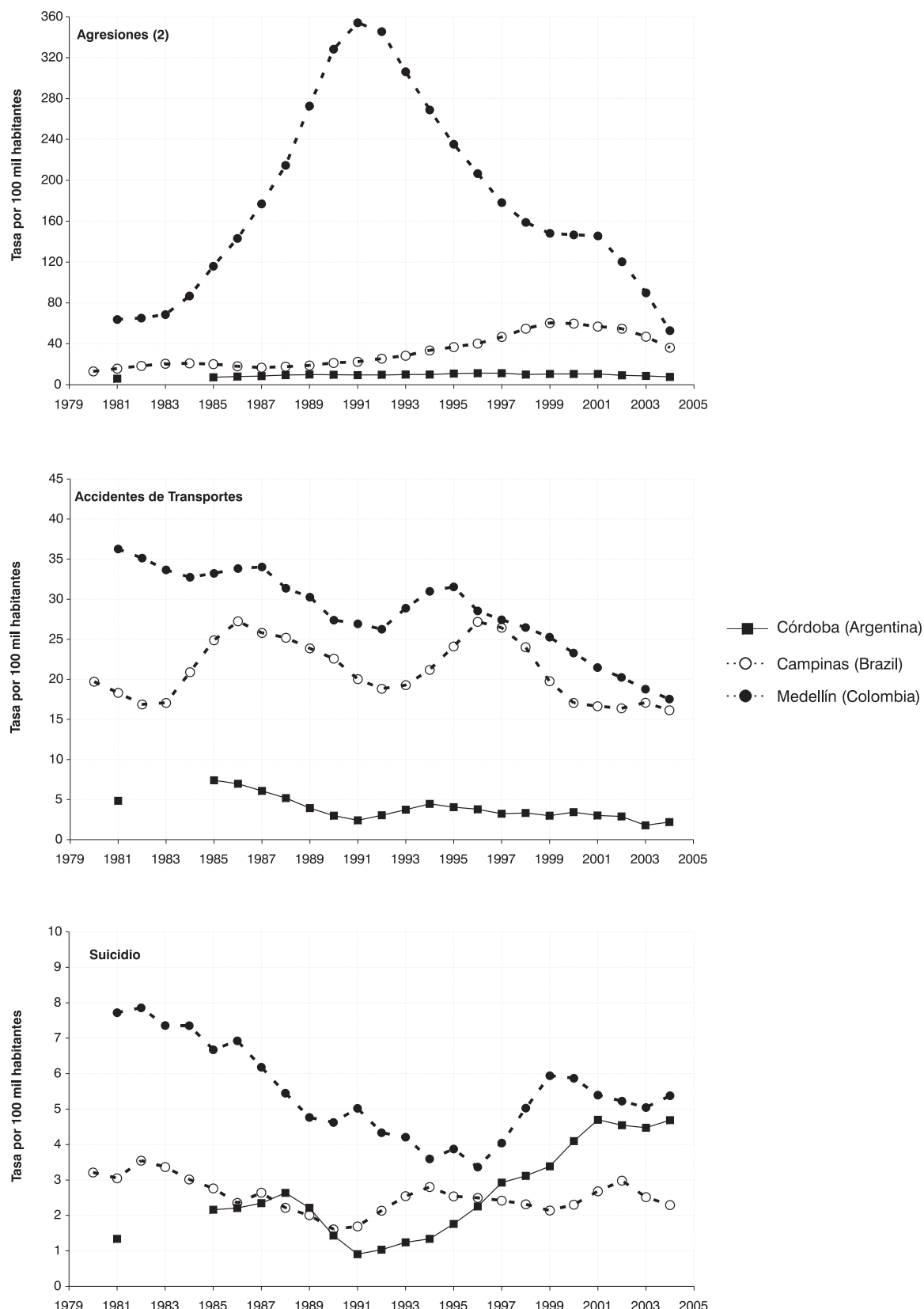

Fuente: IBGE, Censos Nacionales de Brasil; Sistema de Informaciones de Mortalidad, Ministerio de la Salud de Brasil. INDEC, Censos Nacionales de Población de Argentina. Dirección Nacional de Estadísticas e Información en Salud, Ministerio de Salud de Argentina. DANE, Censos Nacionales de Población y Registros de Defunciones de Colombia. Elaboración propia.

(1) Medias trienales.

(2) Todos los homicidios, los accidentes con arma de fuego y otros eventos con arma de fuego con intención no determinada. 
FIGURA 2

Sobremortalidad masculina, según causas externas y trienios seleccionadas Córdoba (Argentina), Campinas (Brasil) y Medellín (Colombia) 1990-2005

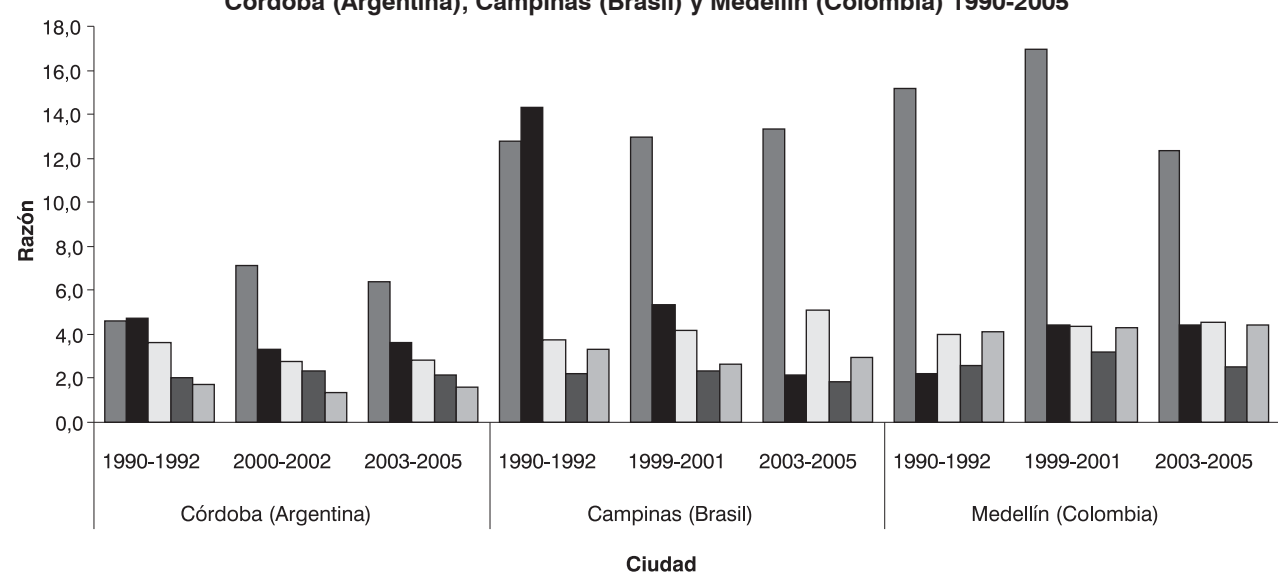

$\square$ Agresiones $\square$ Suicidios $\square$ Acc. de Transportes $\square$ Acc. y otros ev. no det. $\square$ Otras externas

Fuente: IBGE, Censos Nacionales de Brasil; Sistema de Informaciones de Mortalidad, Ministerio de la Salud de Brasil. INDEC, Censos Nacionales de Población de Argentina. Dirección Nacional de Estadísticas e Información en Salud, Ministerio de Salud de Argentina. DANE, Censos Nacionales de Población y Registros de Defunciones de Colombia. Elaboración propia.

la sobremortalidad masculina. En las tres ciudades, el indicador expresa una mayor mortalidad masculina en los cinco grupos de causas externas considerados (Figura 2).

Los cocientes más importantes se aprecian a propósito de las agresiones, en una relación casi directa con el nivel de mortalidad por esta causa. Córdoba y Medellín registran su máximo entre los años 19992001: 7 y 17 fallecimientos de hombres por mujer de cada ciudad, respectivamente. En Campinas, el cociente de sobremortalidad masculina por agresión tiende a mantenerse en 13.

La sobremortalidad masculina por suicidio se reduce drásticamente en Campinas (de 14 a 2), y levemente en Córdoba (desde 5 a 4). En Medellín se aprecia un incremento inicial, con posterior estabilidad en el tiempo (partiendo de 2 en 1990-1992, a prácticamente 5 desde 1999). Aunque las variaciones pudieron ser consecuencia también de la aleatoriedad, por ser eventos más raros, hacia el final del periodo considerado, la razón presenta niveles similares en las tres ciudades.

En las causas restantes (accidentes de transporte, accidentes y eventos no determinados, y otras causas externas), las razones de sobremortalidad masculina nunca son superiores a 5 , y presentan pequeñas variaciones con el paso del tiempo. Puede señalarse entonces que la mortalidad por agresiones y suicidio muestra un patrón prácticamente común a las tres ciudades: los mayores niveles de sobremortalidad masculina.

Concentrando la atención en la distribución por edad de la causa de mortalidad externa más relevante; las agresiones, pueden establecerse nuevas diferencias y similitudes entre las ciudades estudiadas. Como permiten apreciar la Figura 3 , se advierte que las tasas de Medellín superan a las observadas en Campinas y Córdoba, con raras excepciones, en todas las edades y periodos analizados. Esto se comprueba tanto en hombres como en mujeres (aunque los niveles de mortalidad sean considerablemente más altos en los primeros). Por ejemplo, entre los años 1990-1992, se llega a distinguir que a los 20-24 años la tasa de mortalidad por agresión de los hombres de Medellín (1937,4 por cien mil), es 19 veces más alta que su equivalente en Campinas (104,5 por cien mil), y prácticamente 100 
veces más elevada que la correspondiente a Córdoba (19,5 por cien mil). Para la misma fecha y edad, ocurre algo similar en la tasa femenina de mortalidad por agresión: el indicador de Medellín supera 16 veces al de Campinas y 29 veces al de Córdoba (tasas de 104,5 por cien mil; 6,7 por cien mil y 3,6 por cien mil respectivamente).

En cuanto a la distribución de las tasas por agresiones según edad, se aprecia en general: un primer y muy notorio incremento de las tasas entre los 15-19 años, máximo

FIGURA 3

Tasa de mortalidad por agresiones (1) por edad y sexo

Córdoba (Argentina), Campinas (Brasil) y Medellín (Colombia), 1991-2004
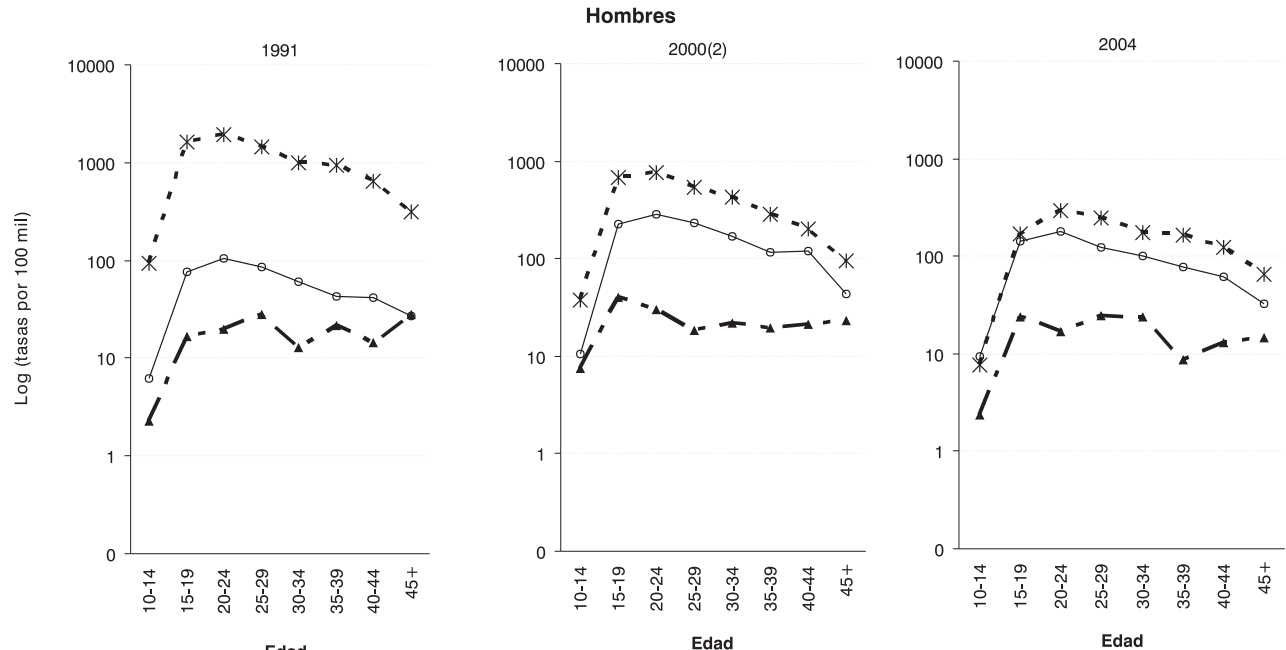

Edad

Mujeres

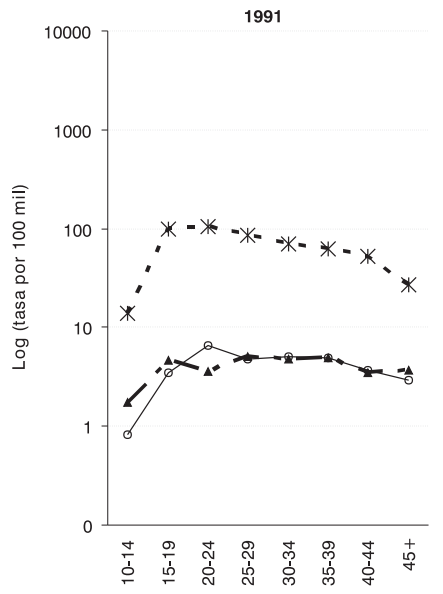

Edad

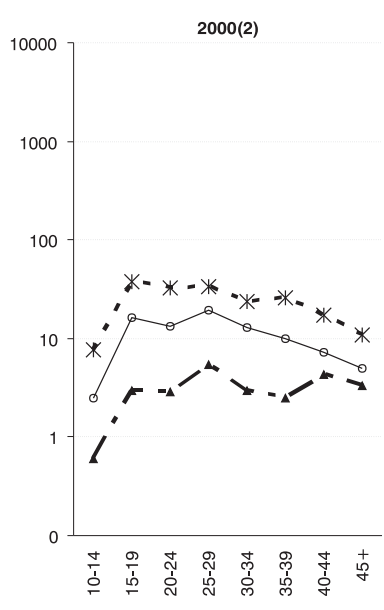

Edad

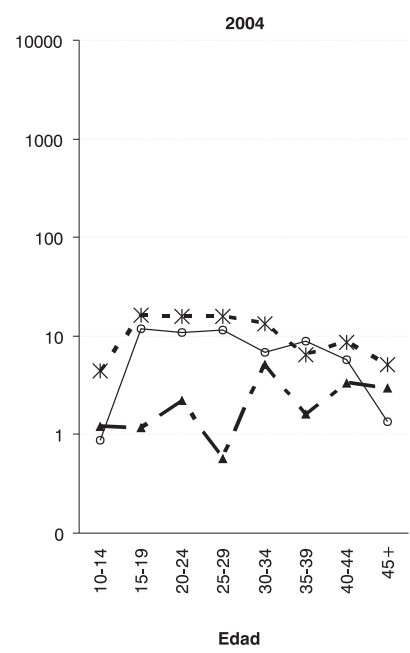

Fuente: IBGE, Censos Nacionales de Brasil; Sistema de Informaciones de Mortalidad, Ministerio de la Salud de Brasil. INDEC Censos Nacionales de Población de Argentina. Dirección Nacional de Estadísticas e Información en Salud, Ministerio de Salud de Argentina. DANE, Censos Nacionales de Población y Registros de Defunciones de Colombia. Elaboración propia.

(1) Todos los homicidios, los accidentes con arma de fuego y otros eventos con arma de fuego con intención no determinada. Medias trienales.

(2) Para Córdoba la tasa se refiere a media de los años 2000, 2001 e 2002. 
a los 20-24 y descenso progresivo a partir de los 25 años. Este patrón en "cúspide" de las tasas de mortalidad por agresiones, es más notorio entre los hombres, y sobre todo en las ciudades de Medellín y Campinas. En Córdoba este comportamiento es mas evidente solamente cerca del año 2000, cuando las tasas son mas altas.

Para el análisis de la distribución por edad de la mortalidad por suicidio, se calcularon tasas promedios para la totalidad del periodo 1990-2005. La Figura 4 muestra un patrón por edad diferente al observado en la mortalidad por agresiones, y a su vez, más diferencial por sexo. Con relación a los hombres, se aprecia que una vez alcanzado un máximo a la edad de 20-24 años, las tasas tienden a mantenerse en niveles similares, reduciéndose en la ciudad que posee los niveles más altos (Medellín), y subiendo en las que registran una mortalidad por suicidio menor (particularmente en Córdoba).

Por el contrario, en las mujeres de Medellín y Córdoba, el máximo de mortalidad por suicidio acontece más tempranamente (15-19 años), para descender entre los 20-24 años y luego mantenerse en los siguientes tramos de edad. Esta constante no se cumple en Campinas, donde la distribución por edad de las tasas femeninas por suicidio, presenta una distribución más parecida a la de los hombres de la misma ciudad.

\section{Discusión}

A escala mundial, se observan diferentes etapas en la capacidad de recopilación de datos. Esta circunstancia incide particularmente en el estudio comparativo de la violencia entre regiones de un mismo país o entre países, ya que afecta la disponibilidad, calidad y comparabilidad de las fuentes de información (OPS, 2002).

En este contexto, es corriente que el estudio de la violencia a través de información sobre mortalidad resulte más sencillo, ya que prácticamente todos los países poseen sistemas de registro de hechos vitales que recogen este dato y los publican oportunamente (OPS, 2002). Además, muchos países disponen de datos de población, que combinados con los datos de defunciones, hacen posible la elaboración de tasas específicas de mortalidad por causas externas. Esta constituye una de las principales ventajas en el uso de estadísticas vitales para el estudio de la violencia.

En cuanto a la calidad de las fuentes de datos, es corriente distinguir dos componentes, que hacen alusión al error de cobertura

FIGURA 4

Tasa de mortalidad por suicidios (1), por edad y sexo

Córdoba (Argentina), Campinas (Brasil) y Medellín (Colombia) - 1990-2005

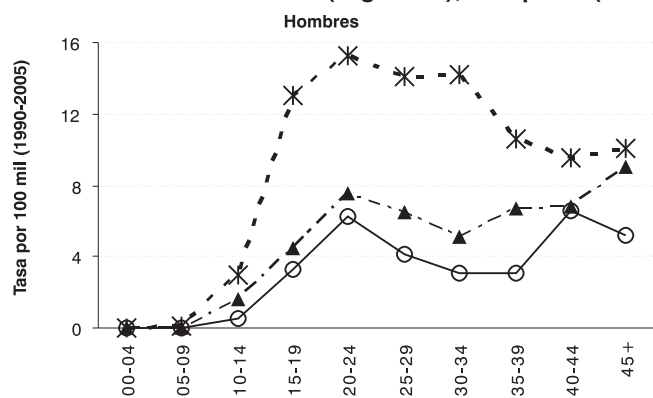

Edad

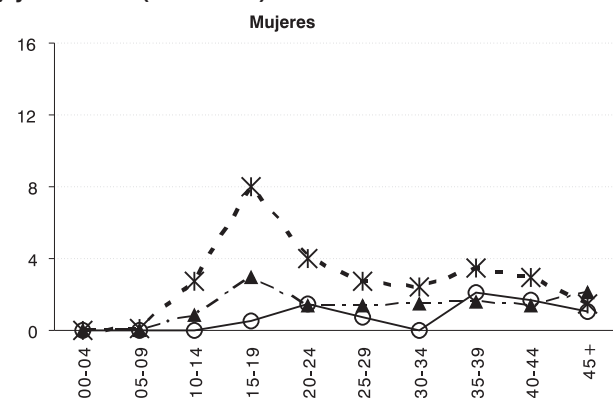

Edad

$$
\text { - - - Córdoba - O Campinas - - - Medellín }
$$

Fuente: IBGE, Censos Nacionales de Brasil; Sistema de Informaciones de Mortalidad, Ministerio de la Salud de Brasil. INDEC Censos Nacionales de Población de Argentina. Dirección Nacional de Estadísticas e Información en Salud, Ministerio de Salud de Argentina. DANE, Censos Nacionales de Población y Registros de Defunciones de Colombia. Elaboración propia.

(1) Promedio de las tasas de mortalidad por suicidios registradas entre 1990-1992, 1999-2001 y 2003-2005. En la ciudad de Córdoba, el segundo periodo corresponde en realidad a 2000-2002. 
y al error de contenido. En la clasificación de Spiegelman (1972 apud CHACKIEL; MACCIÓ, 1978, p.12), los errores de cobertura se refieren a la falta de enumeración o registro de las personas, hechos o cosas en las fuentes de datos. Los errores de contenido hacen alusión a la incorrecta recolección de la información sobre las personas, hechos o cosas en las fuentes de datos. ${ }^{3}$

Si bien ambos tipos de errores afectan por igual a la información censal o del registro vital, en el estudio de la violencia es probable que la fuente más afectada corresponda a las estadísticas sobre defunciones. En primer lugar, porque los problemas de cobertura pueden perjudicar el uso de dicha información de dos maneras, sea en el alcance o en la cobertura misma del registro. ${ }^{4}$ En segundo lugar, los datos de defunciones suelen estar más afectados por problemas de comparabilidad, hecho maximizado en el uso de sistemas clasificatorios que pueden tener diferencias de un país a otro, o dentro de un mismo país a lo largo del tiempo (OPS, 2003).

A su vez, posiblemente la comparabilidad constituya el mayor obstáculo en el estudio de la violencia a partir de información sobre mortalidad. En este tipo de información, la comparabilidad de las causas de muerte está, en parte, garantizada por el uso de la Clasificación Internacional de Enfermedades (CIE), particularmente de sus revisiones más recientes, la $\mathrm{CIE} 9$ y 10 (esta última, vigente desde el año 1997 en Argentina, desde 1996 en Brasil y desde el 2001 en Colombia). Sin embargo, las comparaciones realizadas a partir de una u otra revisión de la CIE deben tomarse con precaución (OPS, 2002), fundamentalmente por el grado de detalle de las clasificaciones y el alcance de las reformulaciones de las normas para la selección de las causas básicas de defunción; ${ }^{5}$ y por las variacio- nes en la precisión del diagnóstico de las causas de muerte de un país a otro. Esta última circunstancia afecta incluso a países en donde la certificación es realizada por un médico, y puede apreciarse en la importancia del porcentaje de defunciones atribuidas a causas de muerte desconocidas o mal definidas. Para las causas externas las diferencias entre las ultimas revisiones se encuentran exclusivamente en la ampliación de los detalles de las circunstancias de los eventos (LAURENTI, 1997).

Las ciudades consideradas en este trabajo son coincidentes en cuanto al estado de la información sobre mortalidad a través de estadísticas vitales. En lo referido a la disponibilidad, corresponden a países que disponen de información sobre causas de muerte de manera temprana, para el contexto regional; Argentina a partir de 1966, Colombia desde 1973 y Brasil en 1977 (MATHERS et al., 2005).

En cuanto al alcance de las estadísticas de defunción, la Argentina cuenta con un $100 \%$ entre las fechas consideradas (OMS, 2007), circunstancia que puede asociarse a la existencia de legislación específica que establece la universalidad del registro civil de las defunciones.

En Colombia, en 1998 se implantó al nivel nacional el Sistema de Registro Civil y Estadísticas Vitales, administrado por el DANE; este sistema unificó el registro de las muertes mediante el Certificado Único de Defunción que mejoró la estructura del formulario (GÓMEZ, 2006). Se desconoce el efecto que estas variaciones hayan tenido sobre la cobertura del registro y la tendencia de las series de datos. Varios estudios sugieren que a lo largo del tiempo, la calidad de los datos de mortalidad ha venido mejorando en Colombia pero los análisis son menos concluyentes en relación con el subregistro. Para 1985 el subregistro de las defunciones

\footnotetext{
${ }^{3}$ En las estadísticas vitales, factores tales como la falta de disponibilidad y de puntualidad son fuentes de error, pero su evaluación no es indispensable a los fines de establecer la calidad de los datos (UNITED NATIONS, 2001, p. 82).

4 OMS (2007) define al alcance de las estadísticas de defunción como el porcentaje de todas las muertes que son registradas en la población cubierta por el registro vital de un país, y a la cobertura; como el cociente entre el total de muertes de un país, reportadas en un año por el sistema de registro de hechos vitales, por el total de muertes estimadas para esa población en el mismo año.

5 En tal sentido, la CIE 10 cuenta con 10.000 condiciones, mientras que la CIE 9 de 5.100. Para las causas externas, la CIE 10 cuenta con 373 condiciones de las causas y la CIE 9 con 192 (LAURENTI, 1997).
} 
podía ascender al 24\%, entre 1987 y 1990 podía haber descendido al 10,0\%. Después de 1998 pudieron haber ejercido un efecto favorable sobre la confiabilidad de la información recolectada y las defunciones ocurridas en centros hospitalarios, no necesariamente sobre la cobertura del sistema (GÓMEZ, 2006). La proporción de causas mal definidas es un indicador de la calidad del registro que ha venido mejorando lentamente en el país; en 1981 se estimaba en 7,5\%; en 1985 descendió a 5,4\% y en 1990 oscilaba alrededor del 3,9\%. La clasificación de la causa de muerte pudo haber mejorado de manera importante partir de 1998, como consecuencia de las mejoras en el certificado de defunción (GÓMEZ, 2006).

En la mortalidad por causas externas es común que intervengan diferentes instancias y por esta razón el subregistro es mucho menor, específicamente las generadas en accidentes y agresiones; por tanto, la omisión encontrada ocurre principalmente a expensas de las muertes por causas naturales.

La provincia y ciudad de Córdoba presenta un porcentaje del $100 \%$ de cobertura ya desde la década del '90 (junto con las provincias de Buenos Aires, Santa Fe, Mendoza y la ciudad Autónoma de Buenos Aires). En Brasil los niveles de la cobertura se incrementan en las áreas más urbanizadas (PAES, 2005). Según informaciones del Instituto Brasilero de Geografía y Estadística (IBGE), y del Ministerio de la Salud, para Sao Paulo la cobertura de los registros alcanza el $100 \%$ en todo el Estado, desde el inicio de los 1990.

El esfuerzo realizado para mostrar el comportamiento de las causas violentas en tres ciudades de América Latina, es un aporte al estudio de las causas específicas de mortalidad que viven los países de la región, puesto que un análisis comparativo requiere de la disponibilidad de fuentes de información confiables, con alcance y con calidad en su recolección, diligenciamiento, tratamiento y transformación.

El análisis señala que Medellín registra una mejor calidad de la clasificación de las causas externas de muerte y que en las otras dos ciudades puede ser mejorada, principal- mente en Córdoba, en donde ha crecido el porcentaje de causas externas por intención no determinada. Al analizar la posibilidad de comparación de los datos se observa que no se encuentran inconvenientes para comparar grupos de causas agregados, ni en la disponibilidad de información, aunque, sí se encuentran plausibles diferencias en algunas causas simples que pueden atribuirse a diferencias en los registros, como es el caso de elevadas cifras de accidentes con armas de fuego y accidentes no determinados en Córdoba y la elevada cifra de eventos de intención no determinada en Campinas.

Las defunciones por causas simples son afectadas por las elevadas cifras de muertes por causas externas mal definidas, que debilitan las conclusiones y requieren de un esfuerzo adicional para poder homologar las comparaciones. Por tal motivo puede señalarse que una de las principales ventajas en el uso de estadísticas vitales para el estudio de la mortalidad por causas violentas es la disponibilidad de información y la homogeneidad por el uso de la Décima Revisión de la Clasificación Internacional de Enfermedades.

Merece destacarse asimismo la alta proporción de causas externas indeterminadas en los registros de Córdoba y Campinas: $45,6 \%$ y $17,5 \%$ en lo trienio $2003 / 2005$, respectivamente. Estas cifras no alcanzan a afectar las comparaciones entre las tres ciudades, ya que las tasas de mortalidad por todas las causas externas presentan patrones muy diferenciados (Figura $1 \mathrm{y}$ Cuadro 3). Sin embargo, para profundizar el conocimiento sobre las características y riesgos de mortalidad por homicidios, suicidios y accidentes de tránsito para la población de Córdoba y Campinas, es necesario que Salud Pública y el Sistema de Seguridad trabajen en el mejoramiento de estos registros.

En general, los niveles de Medellín aventajan notablemente los de Campinas y Córdoba, en todas las causas estudiadas: agresiones, accidentes de transportes, suicidios y otras causas, y en todas ellas son los hombres jóvenes los que ponen la mayor cuota de muertes. Los niveles de Campinas duplican a los de Córdoba, sobre todo en los 
homicidios y accidentes de tránsito en hombres jóvenes, en los que llegan a quintuplicar las cifras cordobesas. Esto no ocurre en los suicidios en donde las tasas cordobesas duplican a las de Campinas.

Los resultados son coherentes con estudios anteriores como en el caso brasilero (AIDAR, 2003), se revelan un crecimiento de las tasas de defunciones por homicidios, incluso en el caso de las mujeres, transformando el perfil de la mortalidad para la población urbana a finales de la década del 1990. En la ciudad de Campinas, en año 2000, los hombres perdieron en general 4,4 años de expectativa de vida por causas violentas y las mujeres 0,92 años. Como en todo lo estado de Sao Paulo, afortunadamente en la región e ciudad de Campinas las muertes por homicidios están diminuyendo desde lo inicio dos años 2000.

La ciudad de Medellín presenta altos índices de violencia, convirtiéndose ésta en la primera causa de muerte de los últimos 20 años. Entre 1994-2003, la población masculina de 20 a 44 años aportó a las causas externas ocasionadas por agresiones con arma de fuego una tasa de 464 por cien mil habitantes; con arma cortopunzante de 44 por cien mil; y por traumatismos por accidentes de transporte de 34 por cien mil; las mujeres del mismo grupo poblacional también aportaron muertes relacionadas con causas externas, principalmente por agresiones con arma de fuego, tasa de 22 por cien mil y accidentes de transporte, 4 por cien mil (CARDONA y AGUDELO, 2006).

Además de los más bajos niveles, la distribución por edad de la mortalidad por causas violentas en Córdoba no es excesivamente concentrada entre los más jóvenes, como en las otras. Este perfil diferenciado ocurre principalmente entre las agresiones y los accidentes de transportes, que son las principales causas de muertes entre los jóvenes de la ciudad brasilera y de la ciudad colombiana. Por otro lado, para la ciudad argentina, los "Accidentes y otros eventos no determinados" acusan la mayoría de las causas violentas.

El comportamiento de la mortalidad por causas externas viene disminuyendo en los tres centros urbanos estudiados, sin diferencias muy elevadas por edad y sexo, y es evidente el bajo comportamiento de las muertes por causas externas en las tres ciudades en los últimos años, que ameritan un análisis de causalidad, de éstas frente al total de las defunciones y por diferentes grupos de causas y desde otras fuentes de información, como registros judiciales y policiales.

Si bien la violencia urbana, estrechamente vinculada con el tráfico de drogas y armas, es un tema de alcance mundial, y para enfrentarla es necesario tener en cuenta las especificidades locales y regionales. ${ }^{6}$ Como revelan los resultados de esta investigación, la tarea no es imposible. Medellín es, sin duda, un caso muy particular a causa de la violencia civil y de su importancia internacional en la producción y distribución de drogas. En este caso, la reducción de la violencia es consecuencia de una serie de acciones que incluyen la desmovilización de los combatientes a cambio de reducciones de penas, una vigorosa ofensiva militar contra la guerrilla, la mejoría en los equipamientos y la renovación de la policía e importantes inversiones sociales en los barrios más pobres de la ciudad. ${ }^{7}$ En Campinas, también indicada como una importante ciudad de consumo y distribución de drogas (ZALUAR, 1999), la reciente reducción de homicidios se ha atribuido, así como para la provincia de Sao Paulo, a la campaña de desarme, seguida también por el mejoría de los equipamientos de la policía y una mayor represión, incluido el crecimiento de los arrestos y detenciones (BOLETIM DE MORTALIDADE, 2005). Otra cuestión que merece discusión es el impacto de los proyectos locales de inclusión social de los

\footnotetext{
${ }^{6}$ Ivan Darío Ramírez, en entrevista del diario “Estadão”, de 6 agosto de 2008. http://www.estado.com.br/editorias/2008/08/06/ pol-1.93.11.20080806.24.1.xml (Acesso em 28/10/2008).

${ }^{7}$ Ruth Costa, en materia del diario "Estadão", de 23 de Março de 2008. http://www.estadao.com.br/estadaodehoje/20080323/ not_imp144459,0.php (Acesso em 28/10/2008).
} 
jóvenes, para la deseada consolidación de la tendencia de disminución en los homicidios en los grandes centros urbanos.

Por último, cabe destacar la importancia de los indicadores de salud para el

\section{Referências bibliográficas}

ADORNO, S. E.; CARDIA, N. Violência, crime, insegurança: há saídas possíveis? In: FONSECA, R. B. et al. (Orgs.). Livro verde: desafios para a gestão da Região Metropolitana de Campinas. Campinas: Instituto de Economia da Unicamp, 2002.

AIDAR, T.; SOARES, M. Desigualdade, vulnerabilidade social e a mortalidade por causas violentas no contexto metropolitano: o caso de Campinas, Brasil. In: CUNHA, J. M. P. (Org.). Novas metrópoles paulistas: população, vulnerabilidade e segregação. Campinas: Nepo/Unicamp, 2006.

AIDAR, T. A face perversa da cidade: configuração sócio-espacial das mortes violentas em Campinas nos anos 90. Textos NEPO 44, Campinas, Nepo/Unicamp, 2003.

ALVAREZ, M. F. La mortalidad por causas externas un desafío multisectorial. In: JORNADAS REGIONALES DE AEPA, 1., 2002. Anais... Córdoba: AEPA, 2002.

BOLETIM DE MORTALIDADE n 37 Mortalidade por homicídios, Coordenadoria de Informação e Informática/SMS/Campinas, Centro Colaborador/DMPS/UNICAMP, Campinas, julho a dezembro de 2005.

CARDONA, A. D.; AGUDELO, G. H. La flor de la vida: pensemos en el adulto: aspectos de la calidad de vida de la población adulta. Medellín: Facultad Nacional de Salud Pública, Universidad de Antioquia, 2006.

CHACKIEL, J; MACCIÓ, G. Evaluación y corrección de datos demográficos. Santiago de Chile: CELADE, 1978. (II - El error en las estadísticas demográficas).

COLOMBIA. Departamento Administrativo Nacional de Estadísticas. Censo de población y vivienda Medellín 2005. Bogotá: DANE, 2007. Disponible en: < http:// www.dane.gov.co/>. seguimiento de la evolución de fenómenos como la violencia experimentada en las relaciones sociales, políticos, familiares y en tránsito, por la población de América Latina.

. Ministerio de Salud. Situación de salud en Colombia: indicadores básicos 2001. Bogotá, 2002.

CUNHA, J. M. P.; OLIVEIRA, A. A. B. População e espaço intra-urbano em Campinas. In: HOGAN, D. J. et al. (Org.). Migração e ambiente nas aglomerações urbanas. Campinas: Nepo/Unicamp, 2001.

FREITEZ, A.; ROMERO, D. Comparación de dos países con mayor crecimiento de la mortalidad juvenil por causas violentas: Brasil y Venezuela. In: CONGRESO LATINOAMERICANO DE POBLACIÓN, 1., 2004, Caxambu. Anais... Belo Horizonte: ABEP, 2004.

GÓMEZ, R. D. La mortalidad evitable como indicador de desempeño de la política sanitaria Colombia 1985-2001. Alicante, 2006. Tesis (Doctoral) - Universidad de Alicante, 2006.

LAURENTI, Ruy. Acidentes e violências/ lesões e envenenamentos e a 10 a revisão da Classificação Internacional de Doenças.

Rev. Saúde Pública, São Paulo, v. 31, n. 4, ago. 1997.

MATHERS, C. et al. Counting de dead and what they died from: an assessment of the global status of cause of dead data. Bulletin of the World Health Organization, Geneva, v.83, n.3, 2005.

ORGANIZACIÓN MUNDIAL DE LA SALUD. Completeness and coverage of death registrationdata. Ginebra, 2007. Disponible en: <http://www.who.int/healthinfo/cod/en/ index.html $>$.

Informe sobre la salud en el mundo 2003. Ginebra, 2003.

ORGANIZACIÓN PANAMERICANA DE LA SALUD. Sobre la estimación de tasas 
de mortalidad para países de la Región de las Américas. Boletín Epidemiología, Colombia, v.24, n.4, 2003.

\section{Informe mundial sobre la}

violencia y la salud. Washington, 2002.

PAES, N. Avaliação da cobertura dos registros de óbitos dos estados brasileiros em 2000. Assessment of completeness of death reporting in Brazilian states for the year 2000. Revista Saúde Pública, São Paulo, v.39, n.6, 2005.

PAES, N.; SILVA, L. A. Perfil de la mortalidad por causas externas en Brasil y en las Unidades de la Federación en la década de 80. Papeles de Población, México, v.26, 2000.
SERFATY, E. et al. Mortalidad por causas violentas em adolescentes y jóvenes de $10 \mathrm{a}$ 24 años, Argentina 1991-2000. Vertex, v.14, supl. 2, 2003.

WAISELFISZ, J. J.; ATHIAS, G. Mapa da violência de São Paulo. Brasília: UNESCO; MEC/INEP; Instituto Ayrton Senna, 2005.

ZALUAR, A. Violence related to illegal drugs "Easy Money" and justice in Brazil: 1980-1995. 1999. (Management of Social Transformations - MOST Discussion Paper, n.35). Disponible en: <http://www.unesco. org/most/zaluar.htm $>$.

\section{Resumo}

Mortalidade por causas externas em três cidades latino-americanas: Córdoba (Argentina), Campinas (Brasil) e Medellín (Colômbia), 1980-2005

O objetivo deste artigo é contribuir para os estudos sobre mortalidade por causas violentas na América Latina, através de análise comparativa dos padrões e tendências das mortes por causas externas em três contextos urbanos. Trata-se de estudo descritivo de fonte secundária, utilizando estatísticas vitais de Córdoba (Argentina), Campinas (Brasil) y Medellín (Colômbia) em três períodos entre 1980 e 2005. São priorizados os subgrupos de causas externas homicídios por armas de fogo e outros; acidentes de transportes; suicídios; e eventos cuja intenção é indeterminada -, com os dados desagregados por idade e sexo e taxas calculadas para o ponto médio dos triênios próximos aos anos censitários. Os resultados indicaram que os níveis de Medellín superam os de Campinas e Córdoba para todas as causas externas estudadas, sendo os homens jovens o grupo mais atingido por tais causas. Os níveis de Campinas duplicam os de Córdoba para os homicídios e acidentes de trânsito, mas, para os suicídios, as taxas da cidade argentina são as mais altas. Para Medellín, as taxas máximas se foram verificadas por volta de 1990, ao contrário das demais, em que a tendência é crescente de 1980 a 2000 e decrescente entre 2001 e 2005. A disponibilidade dos dados de boa qualidade sobre mortalidade permite elaborar comparações entre as populações estudadas. A análise da mortalidade por causas externas evidencia diferenças importantes nos níveis e tendências, e menos no comportamento por sexo e idade, apesar de se tratar de cidades com algumas semelhanças, como o fato de constituírem importantes centros universitários e de desenvolvimento industrial de importância regional e para o país. Os resultados sugerem que fatores socioeconômicos e demográficos não são suficientes para explicar as diferenças observadas.

Palavras-chave: Mortalidade. Causas externas. Violência. Qualidade da informação. 


\begin{abstract}
Mortality from external causes in three Latin American cities: Córdoba (Argentina), Campinas (Brazil) and Medellín (Colombia), 1980-2005

Aim: This article has the objective of contributing to studies on mortality from violent causes in Latin America through a comparative analysis of recent patterns and trends in deaths from external causes in three regional urban contexts.

Methods: A descriptive study from secondary sources is presented, using vital statistics from Cordoba (Argentina), Campinas (Brazil) and Medellin (Colombia) during three different periods between 1980 and 2005. The following subgroups of external causes are studied: homicides by firearms and other weapons, traffic accidents, and suicides and deaths with unspecified intentions. The data was disaggregated by age and sex, with rates calculated for the medians of the next three census years.
\end{abstract}

Results: The levels for Medellin are significantly higher than those seen in Campinas and Cordoba for all external causes studied. Young men constitute the group with the highest mortality. The levels in Campinas are twice those seen in Cordoba, especially in homicides and traffic accidents, but the suicide rates of Cordoba are double those in Campinas. For Medellin the rates were highest around 1990, unlike the two other cities where the trend grew between 1980 and 2000 and declined between 2001 and 2005.

Conclusions: The availability of quality data on mortality allows comparisons among the populations studied. When comparing mortality from external causes, considerable differences in the levels and trends can be seen, but there are fewer differences observed on the data for age and sex. These data were collected from cities with similarities, as they are all three important university centers with industrial development that has been important for their countries. The results suggest that socioeconomic and demographic factors are insufficient to explain the great differences in the data reported.

Keywords: Mortality. External causes. Violence. Quality of information.

Recebido para publicação em 10/10/2008. Aceito para publicação em 14/11/2008. 